\title{
Prediction or hydraulic performance of shell-and-tube heat exchanger: comparison of 1D and CFD-porous media approaches
}

\author{
Loïck Kalioudjoglou, ${ }^{1,2,}$, Clément Bonneau ${ }^{1}$, Vincent Melot $^{1}$, Bruno Auvity ${ }^{2}$, Christophe Josset ${ }^{2}$, and Yoann Merriaux ${ }^{1}$ \\ ${ }^{1}$ Naval Group Indret, 44620 La Montagne, France \\ ${ }^{2}$ Laboratoire de Thermique et Energie de Nantes (LTeN) 3 rue Christian Pauc, 44306 Nantes, France
}

\begin{abstract}
The present paper deals with Heat Exchanger sizing methods and offers a comparison between two of them: 1D global method and CFD porous media method. Following Prithiviraj et al. work [1], new developments are based on recent knowledge acquired on porous media, using a coupling strategy of a three-dimensional commercial code with an in-house code library. The distributed hydraulic resistance concept and the numerical model are briefly described and confronted with pressure drop measurements from an experimental E-type STHE setup (shell-and-tube heat exchanger) from the literature. The present paper will put into perspective capabilities and limits of each method with needs for heat exchanger rating. Flow rate repartition is calculated with CFD-porous media using Tinker's current approach. This new analysis provides a complete comparison with $1 \mathrm{D}$ global method. It also reveals the major impact of leakage flow rate between baffle and tubes. The numerical estimation of pressure losses, consistent with experimental measurements of Halle et al. [2], implies that our future work will include thermal performance characterization and geometrical optimization.
\end{abstract}

\section{Introduction}

In the context of worldwide energy transition, the enhancement of electricity production systems plays a key role. Thus, the optimization of these systems, or of their constitutive components, aims at improving their efficiency, in order to better convert an energy source into electricity. In particular, it covers the complete Rankine cycle and the two-phase heat exchangers, condensers and steam generators. Because of common points with two-phase heat-exchanger flows, a complete understanding of monophasic shell-and-tube heat exchanger fluid dynamic is a preliminary step.

In monophasic STHE, reducing bypass and homogenizing shell side flow contributes to maximizing heat transfer which justify the importance of correct flow predictions. The interdependence between geometrical dimensions, fluid properties and flow involves complex calculations of thermal and hydraulic performances which still remain a challenge.

In order to correctly size heat exchangers, experimental mockup or computational methods may be used: 1D global method, CFD-porous media approach and whole CFD model. The industry sector is increasingly looking for a good compromise between time, cost and accuracy of sizing methods which all have their own benefits and shortcomings.

Experimental mock-up is the most accurate solution but is very costly and slow. Besides, measurements are generally limited to macroscopic measurements.
Numerical models (1D, CFD porous media and whole CFD model) are very cost-saving methods compared to the experimental approach. 1D-global method is the fastest solution with time resolution being a matter of seconds but the unidimensional character of calculation imply sometimes a lack of accuracy. Whole CFD model offers an accurate solution which permit to well understand microscopic local phenomena. However, numerical cost is very important and sometimes models are impossible to use due to a too high mesh number. CFD-porous media is a good compromise between numerical cost and accuracy. It generates a 3D flow field representative of the macroscopic structure within an acceptable time frame and cost.

This paper aims at comparing and criticizing these approaches on an isothermal monophasic shell side water flow case study based on an industrial case introduced by the Argonne National Laboratory [2].

In the tube bundle, baffles are laid-out in order to provide mechanical strength and to mix fluids to improve heat transfers. In practice, clearances between the tube and baffle, and between the baffle and the shell are necessary to assemble the heat exchanger. However, as a result, a leakage occurs through an annular hole. As illustrated in Figure 1 by Tinker [3] the flow could either be transversal or longitudinal to the tube bundle (Bstream), by-pass the bundle (C-stream) or cross the

\footnotetext{
* Corresponding author: loick.kalioudjoglou@naval-group.com
} 
baffles (A and E streams). Consequently, flow structures are strongly heterogeneous and impact the heat transfer distribution. Therefore, the challenge of numerical computation is to correctly evaluate the effects of different streams induced by the position of the tube bundle and the baffle.
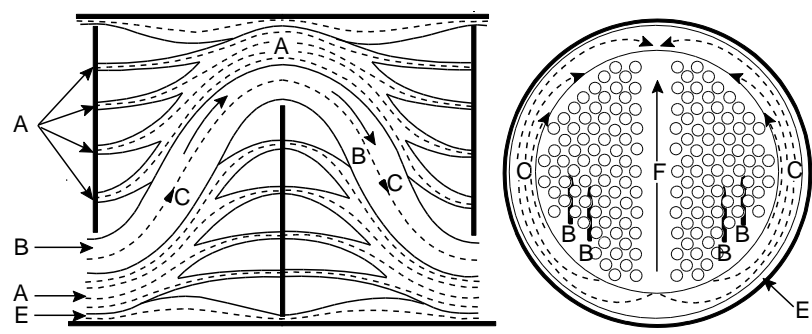

Fig. 1. Flow description through a STHE tube bundle[3].

The challenge of numerical solutions is to correctly estimate the effects of geometry (baffle and geometry layout) on the flow distribution and on pressure losses of shell-and-tube heat exchanger. Therefore, Tinker [3] produced in 1951 a global 1D method which allows to analyze the different current streams aforementioned and to balance the different hydraulic resistance of each one. In this article, a similar iterative global method is used based on Wills and Johnson approach [4].

In 1974, Patankar and Spalding introduced 3D modelling using the first finite difference method [5] with hydraulic resistance distribution model also called porous media model. In this approach, tubes are not simulated in the grid and are replaced by fluid domain considering equivalent volumetric force source terms. This homogenization method permits to know the mean values of velocities and pressures at aimed macroscopic scale and bring a better understanding of local phenomena in STHE with an acceptable calculation cost. Since, Rhodes and Carlucci (1983) [6], Prithiviraj et al. (1998) [1], Vukić et al. (2014) [8], Yang et al. (2014) [7] and Leoni et al. (2017) [9] have applied this approach on finite volume method and improved the accuracy of flow predictions. In this work, a new model, based on a commercial solver is described and compared.

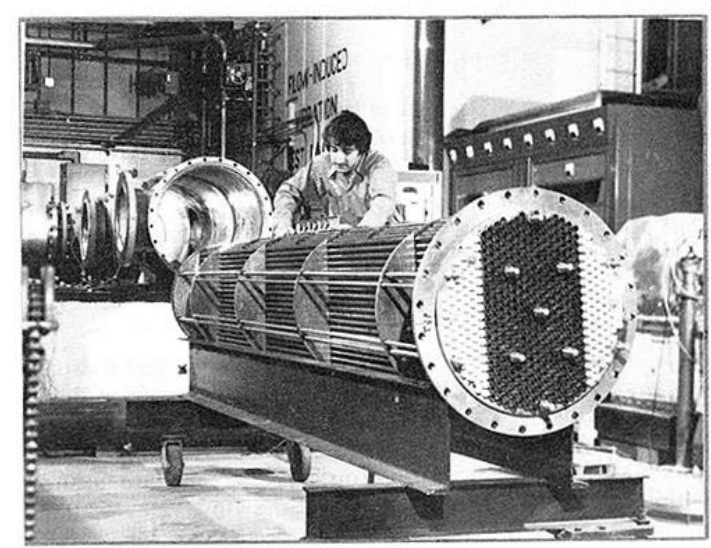

Fig. 2. Test exchanger used by Argonne National Laboratory [2].
More recently, researchers study microscopic flow structure in STHE bundle using CFD simulation applied on real geometries [8], bringing more accuracy on flow prediction. However, these calculations are still applied on small bundle (43 tubes for Heat Exchanger studied by Leoni et al. [8]) because of the limitation of calculation cost.

In this work, a CFD-porous media calculation is solved to better analyze stagnation and recirculation zones and is compared to Halle et al. experiment shown Figure 2. This comparison brings out advantages and shortcomings of all possibilities to sizing heat exchanger.

\section{Analysis and modelling}

\subsection{D-global Method}

A 1-D global method house-code is developed to estimate pressure losses through the shell-side of the heat exchanger with a baffle based on Tinker's stream schematic drawing [3]. With Wills and Johnston method [4], hydraulic resistances are balanced allowing iterative calculation of mass flow rate and pressure losses of each stream.

\subsection{CFD-porous media Method}

A STHE is composed of a regular solid layout (tube bundle) crossed by a heated fluid. The Patankar's concept [5], also called porous media method consists in physically modelling a tube bundle similar to an anisotropic porous media crossed by the flow. Consequently, the media is represented by a continuous medium of liquid and solid.

Considering this homogenization, it is necessary to use empirical laws to describe macroscopic behavior of fluid through bundle. In a numerical way, this media is only fluid and tubes are not represented in the mesh which contains 4.2 million elements. Navier-Stokes equations are modified including source terms to taking account of their presence.

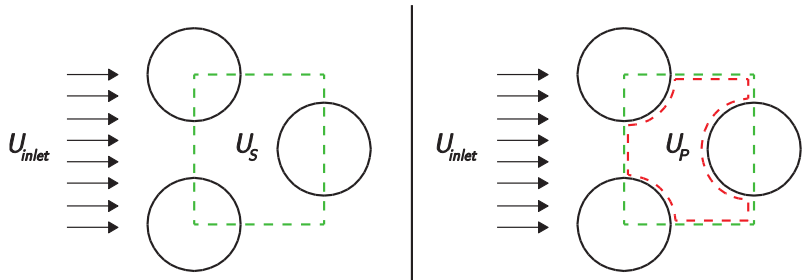

Fig. 3. Superficial velocity (left figure) and physical velocity (right figure) in a tube bundle.

To take account of the tubes presence, fluid porosity is defined as the ratio between fluid volume and total control volume. Superficial velocity is defined as the mean velocity in the porous media Figure 3 illustrated the link between mean physical velocity $U_{p}$ and mean superficial velocitiy $U_{s}$ by porosity value. 


$$
\begin{gathered}
\gamma=\frac{V_{\text {fluid }}}{V_{\text {total }}} \\
U_{s}=\gamma U_{p}
\end{gathered}
$$

Since the objective of this work is not to develop a calculation code, this model is applied on the commercial software ANSYS FLUENT ${ }^{\circledR}$ 16.2. The advection-diffusion equation is written in superficial form in order to keep velocity continuity at the interface between free-domain and porous medium. This method requires a volumetric force term source $\mathrm{F}$ for momentum conservation equation for modelling an equivalent hydraulic resistance. In the bundle, mass and momentum conservation equations are written:

$$
\begin{gathered}
\nabla \cdot\left(\rho \mathbf{U}_{\mathbf{s}}\right)=0 \\
\nabla \cdot\left(\rho \mathbf{U}_{\mathbf{s}} \otimes \mathbf{U}_{\mathbf{s}}\right)=-\nabla \cdot\left(\mu_{\mathrm{eff}} \nabla \mathbf{U}_{\mathbf{s}}\right)-\nabla p+\rho \mathbf{g}+\mathbf{F}
\end{gathered}
$$

Hydraulic resistance is expressed in $\mathrm{N} / \mathrm{m}^{3}$ with $\mathrm{K}$ the pressure drop coefficients tensor.

$$
\mathbf{F}=-\frac{1}{2} \rho \mathbf{K}: \mathbf{U}_{\mathbf{s}}\left\|\mathbf{U}_{\mathbf{s}}\right\|
$$

Considering $\mathrm{Oz}$ the tube axis:

$$
\mathbf{K}=\left(\begin{array}{ccc}
\mathrm{K}_{\mathrm{tr}} & 0 & 0 \\
0 & \mathrm{~K}_{\mathrm{tr}} & 0 \\
0 & 0 & \mathrm{~K}_{\mathrm{lo}}
\end{array}\right)
$$

With $\mathrm{K}_{\mathrm{tr}}$ and $\mathrm{K}_{\mathrm{lo}}$ transversal and longitudinal pressure drops coefficients. These coefficients are calculated with empirical correlations from literature depending on pitch, diameter, pattern and Reynolds number range. A previous study [10] applied on elementary cases highlights the good agreement of empirical laws used by Chalk River Nuclear Laboratories [6][11] for staggered tube bundle.

The longitudinal coefficient is defined in superficial velocity formulation by [11]:

$$
\mathrm{K}_{\mathrm{lo}}=\frac{4 \mathrm{f}_{\mathrm{lo}}}{\mathrm{D}_{\mathrm{h}} \gamma^{2}}
$$

With $\mathrm{D}_{\mathrm{h}}$ and $\mathrm{Re}_{\mathrm{h}}$ the hydraulic diameter and hydraulic Reynolds number defined by :

$$
\begin{gathered}
D_{h}=\left(\frac{2 \sqrt{3}}{\pi}\left(\frac{P}{D_{o}}\right)^{2}-1\right) \times D_{o} \\
\operatorname{Re}_{h}=\frac{\rho U_{p} D_{h}}{\mu}
\end{gathered}
$$

For $\mathrm{Re}_{\mathrm{h}}<2250$ :

$$
f_{l o}=\left(-3.3+22.8 \frac{P}{D_{o}}\right) \operatorname{Re}_{h}^{-1}
$$

For $2250<R \mathrm{e}_{\mathrm{h}}<25000$ :

$$
\mathrm{f}_{\mathrm{lo}}=0.131 \mathrm{Re}_{\mathrm{h}}^{-0.294}
$$

For $\mathrm{Re}_{\mathrm{h}}<25000$ :

$$
\mathrm{f}_{\mathrm{lo}}=0.066 \mathrm{Re}_{\mathrm{h}}^{-0.227}
$$

The transversal coefficient is defined in superficial velocity formulation by [6]:

$$
\mathrm{K}_{\mathrm{tr}}=\frac{4 \mathrm{f}_{\operatorname{tr}}}{\mathrm{P}}\left(\frac{\mathrm{C}_{\mathrm{t}} \mathrm{P}}{\mathrm{P}-\mathrm{D}_{\mathrm{o}}}\right)^{2}
$$

With Re the Reynolds number defined based on the outer diameter :

$$
\operatorname{Re}=\frac{\rho \mathrm{U}_{\mathrm{p}} \mathrm{D}_{\mathrm{o}}}{\mu}
$$

$\operatorname{Re}<8000$

$$
\mathrm{f}_{\mathrm{tr}}=0.619 \mathrm{Re}^{-0.198}
$$

$8000<R e \leq 200000$ :

$$
f_{\text {tr }}=1.156 \operatorname{Re}^{-0.2647}
$$

The transversal coefficient is slightly modified by a coefficient $\mathrm{C}_{t}$ to take into account the fluid attack angle regarding to the bundle orientation as illustrated in Figure 4.

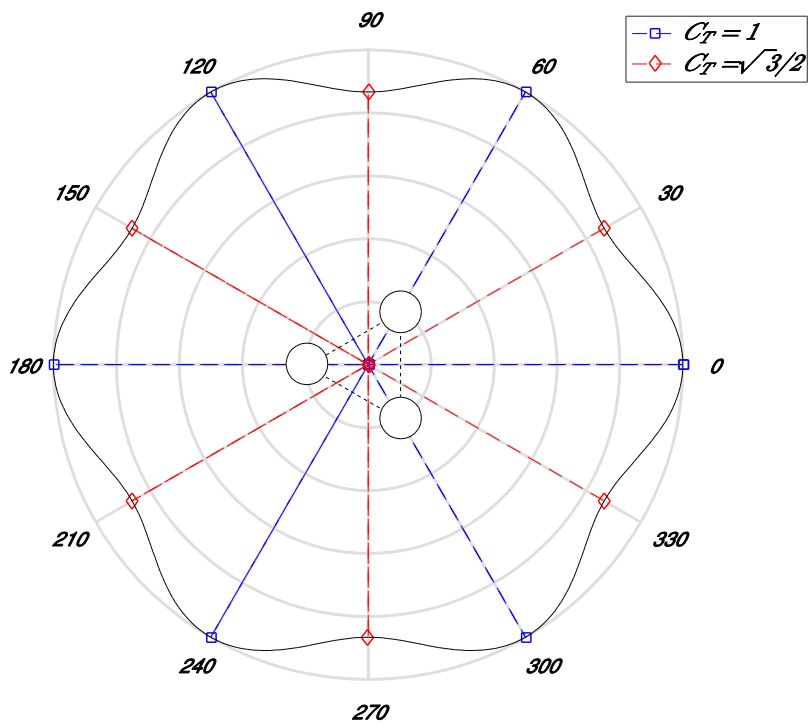

Fig. 4. Variation of coefficient $\mathrm{Ct}$ as a function of attack angle.

$$
C_{t}(\theta)=\frac{1+\frac{\sqrt{3}}{2}}{2}+\frac{1-\frac{\sqrt{3}}{2}}{2} \cos (6 \theta)
$$


Indeed, pressure losses are higher for a nonoriented bundle than for 30-degree oriented bundle due to occurrence of preferable pathways noticeable on Figure 5.

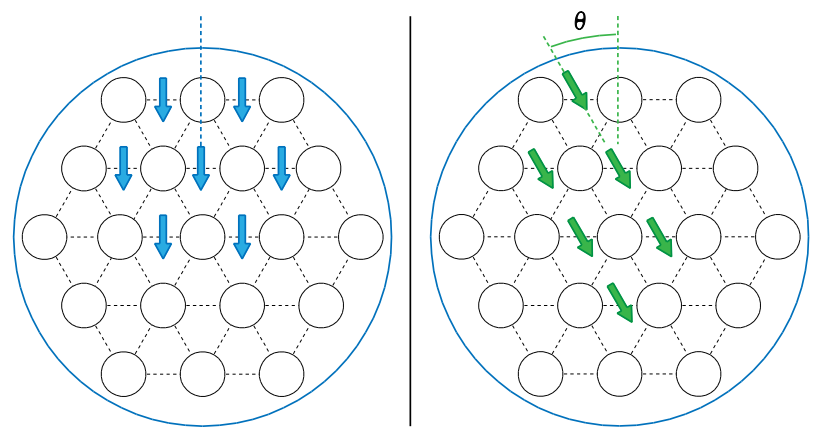

Fig. 5. Non-oriented bundle $\theta=0^{\circ}$ (left figure) and oriented bundle $\theta=30^{\circ}$ (right figure).

Turbulence model for flow in porous media remains a discussed topic in scientific literature. Prithiviraj and Andrews [1] use a $\mathrm{k}-\varepsilon$ turbulent model and consider porous source term to modeling generation of turbulence kinetic energy dissipation rate and generation of turbulence kinetic energy due to tubes. Other authors as Chandresis et al. [12] worked to correctly write this source terms for rod bundle flows. Huang et al. [13] compared calculations made with four different turbulent models in the porous medium. The authors conclude that there are no significant differences between the several calculations on the distribution of velocity and temperature fields.

A sensitivity study was conducted and also showed a very low dependence of heat-exchanger pressure-drop on the turbulent model in the porous medium. In this paper, a $\mathrm{k}-\omega$ SST Turbulence model is used in annular space around heat exchanger tube bundle. However, turbulence model is not used in porous media. Flow in tube bundle is resolved with a laminar model. Indeed, the $\mathrm{K}$ pressure drop coefficient tensor is treated like a catch-all term including turbulence effects. Empirical correlations are based on experimental test results which don't allow isolating tube surface drag term and the Reynolds stress tensor term. Consequently, the use of turbulence model would result in double counting.

Baffles are modelled by a porous media generating leakage rates in tube-to-baffle and shell-to-baffle clearances. Hydraulic resistance is calculated with a pressure drop coefficient tensor using Idel'Cik correlations [14] extended to annular space [15] and constant coefficient $\xi_{\text {in }}=0.5$ et $\xi_{\text {out }}=1$ for specific load losses. This model is both used for tube/baffle and shell/baffle clearance. A sensitivity study depending on baffle's correlation choice has shown a low impact on overall pressure losses.

$$
K=\left(\begin{array}{ccc}
\infty & 0 & 0 \\
0 & \infty & 0 \\
0 & 0 & K_{\text {baffle }}
\end{array}\right)
$$

$$
K_{\text {baffle }}=\left(\frac{\xi_{\text {in }}}{e_{\text {baffle }}}+\frac{f_{\text {baffle }}}{D_{t t b}}+\frac{\xi_{\text {out }}}{e_{\text {baffle }}}\right) \gamma^{2}
$$

\section{Results and discussion}

The considered E-type STHE is a staggered arranged bundle of 499 plain tubes of Halle's experimental test case referenced with the configuration code F.P.6.14" $30^{\circ} \cdot 29 \%$. Table 1 described geometry dimensions and specification. An isothermal water flow is considered with constant properties calculated for a 293.15K temperature. Volumetric flowrate varies between 0.067 and $0.135 \mathrm{~m}^{3} / \mathrm{s}$.

8 pressure taps are disposed at different positions to quantify pressure losses variation in the heat exchanger. For this configuration, overall pressure drop measurement is related to flowrate by Halle et al. in S.I. units with the expression:

$$
\Delta \mathrm{p}=23300\left(\frac{\mathrm{Q}}{0.06309}\right)^{1,87}
$$

Table 1. Dimension and Specification of F $P \cdot 6 \cdot 14 " \cdot 30^{\circ} \cdot 29 \%$

Heat Exchanger of Argonne National Laboratory [2].

\begin{tabular}{|c|c|}
\hline Shell diameter (m) & $\mathbf{0 . 5 9 0}$ \\
\hline Tube length (m) & 3.580 \\
\hline Tube outer diameter (mm) & 19.1 \\
\hline Pitch to diameter ratio (-) & 1.25 \\
\hline Tube numbers (-) & 499 \\
\hline Baffle numbers (-) & 5 \\
\hline Baffle outer diameter (m) & 0.587 \\
\hline $\begin{array}{c}\text { Baffle thickness (mm) } \\
\text { clearance } \mathrm{D}_{\text {ttb }}(\mathrm{mm})\end{array}$ & 9.5 \\
\hline $\begin{array}{c}\text { Nozzle diameter (m) } \\
\text { Minimum tube-baffle hole diametric }\end{array}$ & 0.4 \\
\hline $\begin{array}{c}\text { Baffle cut space as percentage of inside } \\
\text { shell diameter (\%) }\end{array}$ & 28.9 \\
\hline
\end{tabular}

An overall pressure losses comparison between numerical calculations and experimental measurement is shown on Figure 6 and quantified on Table 2. The numerical evolution of pressure losses is also subject to a volumetric flow rate power law similar to experimental observation. For specified tube-to-baffle clearance $\mathrm{D}_{\mathrm{ttb}}=0.4 \mathrm{~mm}$, pressure losses are underestimated by CFD porous media model and overestimated by 1Dglobal method. These values remain within an acceptable range compared to measurement error which can reach $+-5.3 \%$ [2]. However, 1D code show significant 
difference when clearance is not considered. It can be easily noticed that clearance values have a very strong impact on overall pressure drop.

Table 2. Relative deviation between experimental and numerical pressure losses.

\begin{tabular}{|c|ccc|}
\hline & \multicolumn{3}{|c|}{ Relative deviation (\%) } \\
\hline Dttb (mm) & 0 & 0.4 & 0.8 \\
\hline $\begin{array}{c}\text { CFD- } \\
\text { Porous } \\
\text { Media } \\
1 \mathrm{D}\end{array}$ & $+18 \%$ & $-12 \%$ & $-31 \%$ \\
& $+125 \%$ & $+28 \%$ & $+8 \%$ \\
\hline
\end{tabular}

Leoni et al. [9] has already shown impact of clearance for a small 43-tubes shell and tube heat exchanger with important variations between withoutclearance and $0.8 \mathrm{~mm}$-clearance configurations. It shows importance of precise specification of clearance of heat exchanger for a correct sizing.

In practice, despite reduction of pressure drop, presence of large clearance isn't desirable because it creates large decrease of heat exchanges.

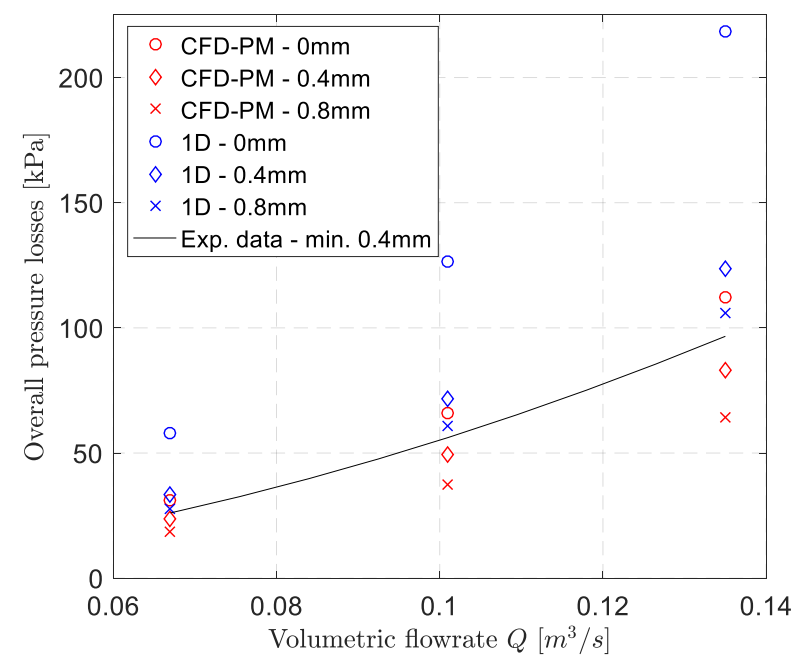

Fig. 6. Comparison of overall pressure losses numerically obtained with experimental measurements.

Figure 7 represents spatial distribution of pressure losses in the STHE. It is obvious that CFD-porous medial model which is three-dimensional allows to record local phenomena like recirculation zones, bypassing or local pressure variation which explain a better agreement compared to 1D-global method.

In addition to pressure losses values, qualitative analysis is a necessary step. An incorrect flow distribution could cause a wrong hydraulic performance prediction. Figure 8 highlights flow structure and local repartition of different streams with streamlines colored by velocity.

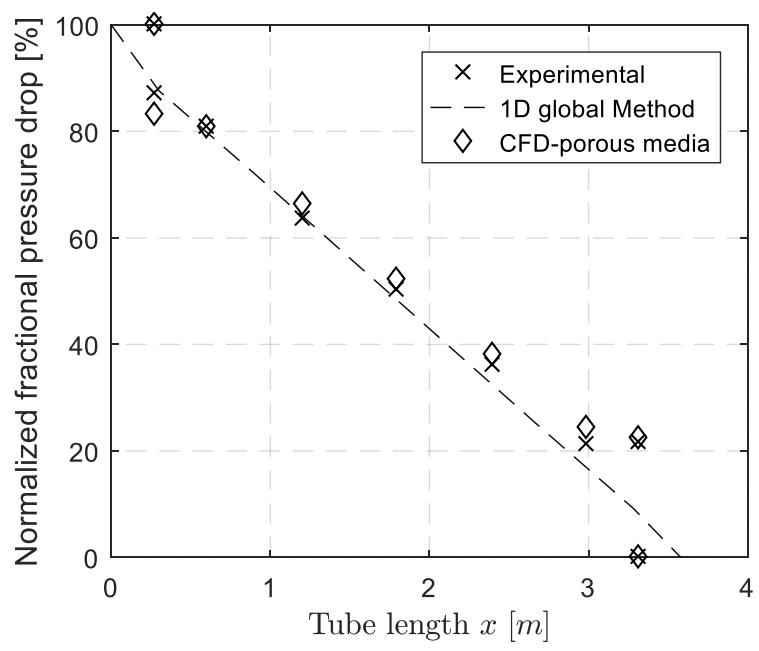

Fig. 7. Fractional distribution of pressure drop averaged and normalized to overall pressure drop for a Volumetric Flow Rate of $\mathrm{Q}=100.8 \mathrm{~m}^{3} / \mathrm{s}$.

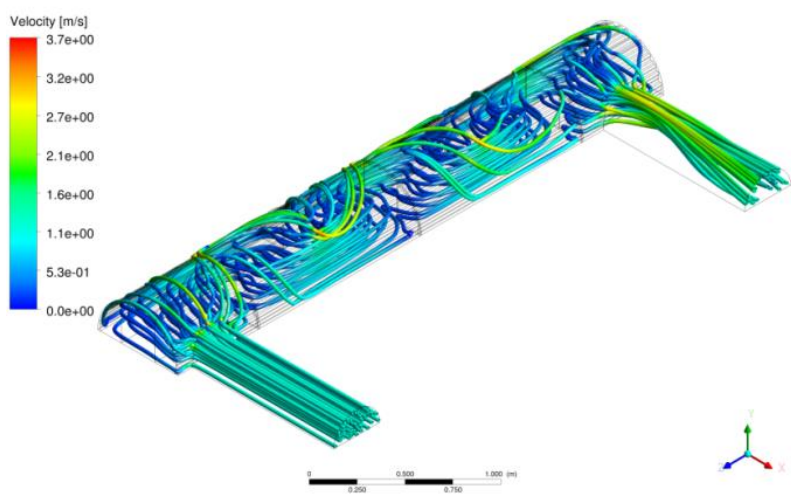

Fig. 8. Streamlines (colored by velocity) in porous media modelled heat exchanger.

Table 3. Tinker's Stream Flow Rate Estimation for a Volumetric Flow Rate of $\mathrm{Q}=100.8 \mathrm{~m}^{3} / \mathrm{s}$.

\begin{tabular}{cccccc|c}
\hline Method & $\begin{array}{c}\mathrm{D}_{\mathrm{ttb}} \\
(\mathrm{mm})\end{array}$ & $\mathrm{A}$ & $\mathrm{B}$ & $\mathrm{C}$ & $\mathrm{E}$ & $\mathrm{Q}_{\mathrm{B}} / \mathrm{Q}_{\mathrm{C}}$ \\
\hline 1D & None & 0 & 87.0 & 13.0 & 0 & 6.7 \\
CFD PM & None & 0 & 76.1 & 23.9 & 0 & 3.2 \\
1D & 0.4 & 7.5 & 74,5 & 10.9 & 7.0 & 6.8 \\
CFD PM & 0.4 & 11.8 & 64.5 & 16.1 & 7.5 & 4.0 \\
1D & 0.8 & 19.0 & 65.4 & 9.4 & 6.2 & 7.0 \\
CFD PM & 0.8 & 26.4 & 53.4 & 13.9 & 6.4 & 3.8 \\
Well & & & $40-$ & $15-$ & $6-$ & $2-4.7$ \\
designed & & $9-20$ & 70 & 20 & 20 & \\
STHE & & & & &
\end{tabular}

It can be shown that the influence of C-stream bypass could decrease strongly the efficiency. A local depression zone can be seen at the outlet. Definitions of plane in post-processing enable numerical calculation of the mass flow rate of each stream. Table 3 compares flow rate repartitions calculated by both methods. These planes are defined on the central crosspass to avoid border effects next to inlet and outlet. 
A-stream mass flow rate growth significantly when tube-to-baffle clearance increases. Nevertheless, flow rate ratio $Q_{B} / Q_{C}$ does not change with clearance and remains constant for each method. A considerable difference exists between C-stream predictions of 1D method and CFD-porous media method. The latter method forecast a larger fluid bypass. Taborek's feedback [16] identifies in Table 3 ranges of welldesigned heat exchanger flow fractions based on industrial size exchangers from the Heat Transfer Research, Inc. data bank. If we only consider results for $0.4 \mathrm{~mm}$ baffle-to-tube clearances, it can be observed that prediction of ratio $Q_{B} / Q_{C}$ with $1 D$ method seem to be slightly superior than experience observations.

Evolution of velocity field plotted on symmetry plane Figure 9 highlights a good distribution of flow : the fluid uses the full depth of the crosspass and the heat exchanger design does not create low-Reynolds recirculation zone.
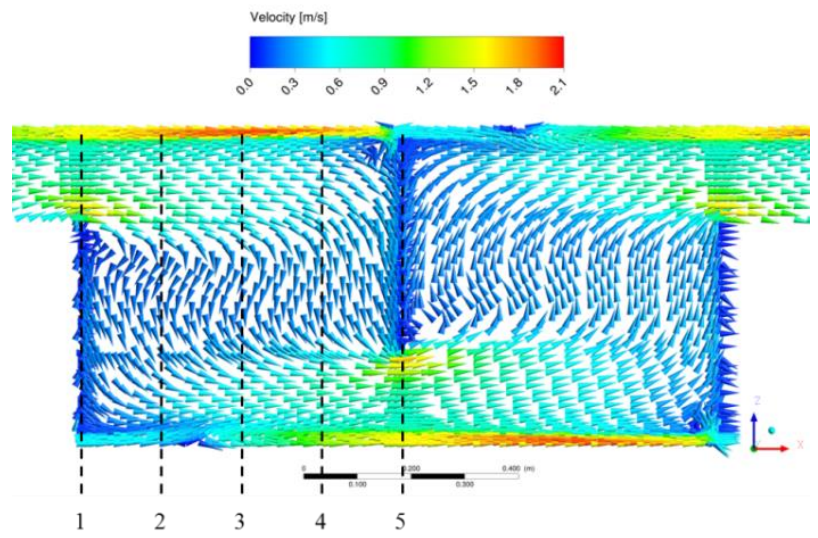

Fig. 9. Velocity field passing through baffle on plane symmetry for a volumetric flow rate of $Q=100.8 \mathrm{~m}^{3} / \mathrm{s}$.

Taborek described in [16] heat exchanger sizing recommendations. He gives optimum zone which relates baffle cut (\%), crosspass depth (m) and shell diameter. For the Halle et al. configuration, the optimal baffle cut value recommandate by Taborek is slightly superior : $36 \%$ while experimental mock-up consider a $28.9 \%$ baffle cut. Based on developed 1D model and CFDporous media model, a parametric study could verify the quality of these sizing advised and improve geometric design.

In order to examine the impact of porous media on flow structure, five velocity fields between second and third baffle have been exported from simulation. The positions of each plane are indicated on Figure 9.

In plane 1 , one portion of fluid crosses the baffle (Astream and E-stream). The other portion, is distinguished in the baffle window between tube bundle and annular space. The more the space between shell and bundle is significant, the more the bypass flow rate is high [10]. It can be noticed that in plane 5, the flow is completely reversed compared to plane 1 and a similar flow is perceptible.
Between planes 1 and 5, the flow crosses (B-stream) and bypasses (C-stream) the bundle to reach the second window. In contrast to STHE studied by Bonneau [10], the flow is not broken when getting closed to baffle but gradually moves towards the second window and provides a better flow distribution and thus a better thermal efficiency.

\section{Conclusions}

The application of different sizing tools was set up. A triple confrontation between experimental measurements, 1D global method and CFD-porous media allowed to secure and make reliable their utilization.

It has been demonstrated that CFD-porous media brings an acceptable accuracy lower than $15 \%$ for specified value of $0.4 \mathrm{~mm}$ by experiments [2]. Nevertheless, the CFD-porous media code tends to underpredict pressure losses which involves the use of safety margins for some industrials processes.

1D global method is very fast and allows to consider optimization loops at low calculation costs. However, accuracy is lower than CFD-porous media and overprediction of pressure drops can go up to $30 \%$ in comparison to experimental measurements.

It has been shown than not considering the clearance can be damaging for sizing with an overprediction of pressure losses which can reach $18 \%$ for CFD-porous media and $125 \%$ for 1D global method.

The strong impact of tube-to-baffle clearance has shown the need to correctly specify the clearance for an accurate pressure drop prediction and as a result a correct thermal prediction. Actually, it is difficult to judge the accuracy of numerical code due to clearance value communicated by Halle \& al. which is not a nominal but a minimal value.

Calculations confirm designer's experience regarding to bypasss impact, baffle geometry and position influence. The code provides an operating tool for verifying by $3 \mathrm{D}$ simulation acquired experiences.

In the future, design optimization study could be considered with sealing strips inserts, bundle geometric adjustments and modifications of positions or shapes of baffles. Besides, design recommendations established by Taborek [16] could be fortified with numerical parametric study.

Finally, a thermal validation study of Shell and Tube Heat Exchanger by CFD porous media would allow to undertake diphasic shell and tube heat exchangers with correct prediction of monophasic cooling and heating regimes.

\section{Acknowledgements}

Thanks are due to Mr. S. DITTIERE for the contribution in the calculation step. The authors gratefully acknowledge the supports of Naval Group and Thermal and Energy laboratory of Nantes (LTeN). 


\section{Nomenclature}

\begin{tabular}{lcc}
\hline Quantity & Symbol & $\begin{array}{c}\text { Coherent } \\
\text { SI Unit }\end{array}$ \\
\hline Fluid orientation coefficient & $\mathrm{C}_{\mathrm{t}}$ & - \\
Bundle Hydraulic Diameter & $\mathrm{D}_{\mathrm{h}}$ & $\mathrm{m}$ \\
Tube to baffle hole & $\mathrm{D}_{\mathrm{ttb}}$ & $\mathrm{m}$ \\
diametral clearance & $\mathrm{D}_{\mathrm{o}}$ & $\mathrm{m}$ \\
Outer diameter & $\mathrm{F}$ & $\mathrm{N} / \mathrm{m}^{3}$ \\
Volumetric Term source & $\mathrm{f}_{\mathrm{lo}}$ & - \\
Longitudinal friction factor & $\mathrm{f}_{\mathrm{tr}}$ & - \\
Transversal friction factor & $\mathrm{K}_{\mathrm{tr}}$ & $\mathrm{m}^{-1}$ \\
Transversal pressure drop & & \\
coefficient & $\mathrm{K}_{\mathrm{lo}}$ & $\mathrm{m}^{-1}$ \\
Longitudinal pressure drop & & \\
coefficient & $\mathrm{K}_{\mathrm{baffle}}$ & $\mathrm{m}^{-1}$ \\
Baffle pressure drop & $\mathrm{P}$ & $\mathrm{m}$ \\
coefficient & $\Delta \mathrm{p}$ & $\mathrm{Pa}$ \\
Pitch & $\mathrm{Q}$ & $\mathrm{m}^{3} / \mathrm{s}$ \\
Pressure drop & $\mathrm{U}_{\mathrm{p}}$ & $\mathrm{m} / \mathrm{s}$ \\
Volumetric flow rate & $\mathrm{U}_{\mathrm{s}}$ & $\mathrm{m} / \mathrm{s}$ \\
Physical velocity & $\gamma$ & - \\
Superficial velocity & $\theta$ & $\mathrm{rad}$ \\
Porosity & $\mu_{\mathrm{eff}}$ & $\mathrm{Pa} . \mathrm{s}$ \\
Attack angle & $\xi$ & - \\
Effective viscosity & & \\
Singular friction factor & & \\
\hline
\end{tabular}

\section{References}

1. M. Prithiviraj and M.J. Andrews, Numerical Heat Transfer, 33, 8, pp. 799-816 (1998)

2. H. Halle, J.H. Chenoweth and M. W. Wambsganss, Journal of heat transfer, 110, 1, pp. 60-67 (1988)

3. T. Tinker, Proceedings of the General Discussion on Heat Transfer, pp. 89-96 (1951)

4. M.J.N. Wills and D. Johnston, 22nd National Heat Transfer Conference, HTD, 36, pp. 67-79 (1984)

5. S.V. Patankar and D.B. Spalding, Heat Exchangers: Design and Theory Source Book (McGraw-Hill, New York, pp. 155-176, 1974)

6. D.B. Rhodes and L.N. Carlucci, Proceedings of Canadian Nuclear Society - American Nuclear Society International Conference on Numerical Methods in Engineering, (Montreal, pp. 935-948, 1983)

7. J. Yang, L. Ma, J. Bock, A.M. Jacobi and W. Liu, Applied Thermal Engineering, 65, 1-2, pp. 369-383 (2014)

8. M.V. Vukić, G. Vučković, P., Živković, Ž. Stevanović and M. Tomić, Facta Universitatis, Series: Mechanical Engineering, 11, 2, pp. 169-180 (2014)

9. G.B. Leoni, T.S. Klein and R. De Andrade Medronho, Applied Thermal Engineering, 112, pp. 497-506 (2017)

10. C. Bonneau, Ph.D. dissertation, Nantes University, France (2017)
11. M.B. Carver, L. N. Carlucci and W. W. R. Inch, No. AECL-7254, Atomic Energy of Canada Ltd. (1981)

12. M. Chandresis, G. Serre and P. Sagaut, International Journal of Heat and Mass Transfer, 49, 15, pp.2739-2750 (2006)

13. L.Y. Huang, J. X. Wen, T. G. Karayiannis, R. D. Mathews, The PHOENICS Journal of Computations Fluid Dynamics \& its applications, 9, 2, pp. 181-209 (1996)

14. I. E. Idel'Cik, Collection de la Direction des Etudes et Recherches d'Electricité de France (Paris, France, 1969)

15. W.R. Van Zyl, J. Dirker and J. P. Meyer, Heat Transfer Engineering, 34, 13, pp 1112-1123 (2013)

16. J. Taborek, Heat Exchanger Design Handbook (1983) 